\title{
More on the Free Factors of Parafree Lie Algebras
}

\author{
İbrahim Halil Coşkun \\ Harran University, Department of Mathematics, Şanlıurfa, Turkey
}

\begin{abstract}
Parafree Lie algebras are algebras that are residually nilpotent and satisfy the property that their quotients by the terms of the lower central series are isomorphic to the corresponding quotients of a free Lie algebra. There are many nonfree parafree Lie algebras and they all have many properties in common with free Lie algebras. In this paper, our main goal is to investigate the free product of parafree Lie algebras. In particular, we prove that a finite number of free factors of a parafree Lie algebra are parafree.
\end{abstract}

Keywords: Free Lie algebras, Parafree Lie algebras, Free Product, Free Factors

\section{INTRODUCTION}

Let $\mathrm{L}$ be a Lie algebra over a field $\mathrm{k}$. The series

is called lower central series of $\mathrm{L}$ and defined

$$
\mathrm{L}=\gamma_{1}(L) \supset \gamma_{2}(L) \supset \gamma_{3}(L) \supset \ldots \supset \gamma_{k}(L) \supset \ldots
$$

$$
\gamma_{1}(L)=L, \gamma_{2}(L)=[\mathrm{L}, \mathrm{L}], \gamma_{3}(L)=\left[\mathrm{L}, \gamma_{2}(L)\right], \ldots, \gamma_{k}(L)=\left[\mathrm{L}, \gamma_{k-1}(L)\right], \ldots
$$

If $\mathrm{k}$ is the smallest positive integer that satisfying $\gamma_{\mathrm{k}}(\mathrm{L})=\{0\}$ then $\mathrm{L}$ is called nilpotent of class $\mathrm{k}$. The quotient algebras $\mathrm{L} / \gamma_{2}(\mathrm{~L}), \mathrm{L} / \gamma_{3}(\mathrm{~L}), \mathrm{L} / \gamma_{4}(\mathrm{~L}), \ldots$ are called the lower central sequence of $\mathrm{L}$.

Let $\mathrm{F}$ and $\mathrm{L}$ be two Lie algebras. For all $\mathrm{k} \geq 1$, if we have

$$
\mathrm{L} / \gamma_{k}(\mathrm{~L}) \cong \mathrm{F} / \gamma_{k}(\mathrm{~F})
$$

then we call that " $\mathrm{L}$ and $\mathrm{F}$ have the same lower central sequence".

Let $\mathrm{L}$ be a Lie algebra over a field $\mathrm{k}$. For any $0 \neq \mathrm{gCL}$ if there exists a homomorphism $\psi_{\mathrm{g}}$ form $\mathrm{L}$ to a nilpotent Lie algebra $\mathrm{N}$ such that $\psi_{\mathrm{g}}(\mathrm{g}) \neq 0$ then $\mathrm{L}$ is called residually nilpotent.

For more details about the Lie algebra theory, see [Erdmann and Wildon, 2006; Jacobson, 1979].

A Lie algebra $\mathrm{L}$ is called parafree if it is residually nilpotent and has the same lower central sequence as a free Lie algebra. Let $B \subset L, L \equiv \bar{L}\left(\bmod \gamma_{2}(L)\right)$ and $\equiv \bar{B}\left(\bmod \gamma_{2}(L)\right)$. If $\bar{B}$ freely generates $\bar{L}$, then it is called that " B freely generates L modulo $\gamma_{2}(L)$ '”.

Parafree Lie algebras firstly have defined by Baur (1978). These special Lie algebras satisfy properties that are analogous to those of parafree groups (Baumslag, 1967, 1969). Velioğlu (2013) has proved that a subalgebra and the quotient algebra of a parafree Lie algebra are again parafree. Moreover Ekici and Velioğlu (2014) have studied on unions of parafree Lie algebras and they have proved that the direct limit of a system of parafree Lie algebras is parafree (2015). Velioğlu (2019) has obtained some results about parafree metabelian Lie algebras. Later Ekici and Velioğlu (2019) have investigated the metabelian product of parafree Lie algebras. Despite all these studies, the theory on parafree Lie algebras has still answered questions. We have taken this opportunity to obtain some results about parafree Lie algebras.

The aim of this work is to investigate the free product of parafree Lie algebras. In particular, we prove that a finite number of free factors of a parafree Lie algebra are parafree.

\section{MAIN RESULTS}

Definition 2.1. Let $\left(G_{\alpha}\right)_{\alpha \in I}$ be a family of Lie algebras over a field k with presentations $\left(X_{\alpha} / R_{\alpha}\right)$ where for all $\alpha \neq \beta$ we have $X_{\alpha} \cap X_{\beta}=\emptyset$. Set $X=\bigcup_{\alpha} X_{\alpha}, R=\bigcup_{\alpha} R_{\alpha}$. Then $G=(X, R)$ is called the free product of family $\left(G_{\alpha}\right)_{\alpha \in I}$ and it is denoted by $G=\prod_{\alpha} * G_{\alpha}$.

There is a canonical epimorphism from the free product $\prod_{\alpha} * G_{\alpha}$ to the direct sum $\bigoplus_{\alpha} G_{\alpha}$ of the family $\left(G_{\alpha}\right)_{\alpha \in I}$. This epimorphism is defined by identity map of algebras $\left(G_{\alpha}\right)_{\alpha \in I}$ to theirself.

If $I=\{1,2, \ldots, n\}$, it is simply written

$$
G=G_{1} * G_{2} * \ldots * G_{n}
$$




\section{International Advanced Research Journal in Science, Engineering and Technology}

Vol. 7, Issue 2, February 2020

The algebras $G_{1}, G_{2}, \ldots, G_{n}$ are called the free factors of $\mathrm{G}$. Note that for any $\alpha \in I$ there exists a homomorphism $I_{\alpha}: G_{\alpha} \rightarrow G$, extending the identity map of $X_{\alpha}$ to $X$ (for more details see [Bahturin, 1987]).

Baur 1978 studied on free product of parafree Lie algebras and he proved that the free product of a finite number of parafree Lie algebra is parafree by using homological methods.

Let $R=R_{1} * R_{2}$ be a parafree Lie algebra of two Lie algebras $R_{1}, R_{2}$. Velioğlu 2013 has proved that the free factors $R_{1}, R_{2}$ of parafree Lie algebra $\mathrm{R}$ are parafree. We want to generalise this result by proving it for a finite numbers free factors of a parafree Lie algebra. We carry the formal arguments used in Velioğlu 2013. To do that we need the following propositions.

Proposition 2.1. Let $\mathrm{A}_{1}, \ldots, A_{n}$ be a finite number of parafree Lie algebras. Then we have the following equation.

$\mathrm{A}_{1} / \gamma_{2}\left(\mathrm{~A}_{1}\right) \oplus \cdots \oplus \mathrm{A}_{n} / \gamma_{2}\left(A_{n}\right)=\mathrm{A}_{1} \oplus \cdots \oplus \mathrm{A}_{n} / \gamma_{2}\left(\mathrm{~A}_{1}\right) \oplus \cdots \oplus \gamma_{2}\left(\mathrm{~A}_{n}\right)$

Proof. Consider a element $\left(a_{1}+\gamma_{2}\left(\mathrm{~A}_{1}\right)\right)+\cdots+\left(a_{n}+\gamma_{2}\left(A_{n}\right)\right)$ of the algebra $\mathrm{A}_{1} / \gamma_{2}\left(\mathrm{~A}_{1}\right) \oplus \cdots \oplus \oplus^{\mathrm{A}_{n}} / \gamma_{2}\left(\mathrm{~A}_{n}\right)$.

It is clear that

$\left(a_{1}+\gamma_{2}\left(\mathrm{~A}_{1}\right)\right)+\cdots+\left(a_{n}+\gamma_{2}\left(A_{n}\right)\right)$

$=\left(a_{1}+\cdots+a_{n}+\gamma_{2}\left(\mathrm{~A}_{1}\right)+\cdots+\gamma_{2}\left(A_{n}\right)\right) \in^{\mathrm{A}_{1} \oplus \cdots \oplus \mathrm{A}_{n}} / \gamma_{2}\left(\mathrm{~A}_{1}\right) \oplus \cdots \oplus \gamma_{2}\left(\mathrm{~A}_{n}\right)$.

On the other hand, for any element $\left(a_{1}+\cdots+a_{n}+\gamma_{2}\left(\mathrm{~A}_{1}\right)+\cdots+\gamma_{2}\left(A_{n}\right)\right)$ of the algebra

$\mathrm{A}_{1} \oplus \cdots \oplus \mathrm{A}_{n} / \gamma_{2}\left(\mathrm{~A}_{1}\right) \oplus \cdots \oplus \gamma_{2}\left(\mathrm{~A}_{n}\right)$, we have

$\left(a_{1}+\cdots+a_{n}+\gamma_{2}\left(\mathrm{~A}_{1}\right)+\cdots+\gamma_{2}\left(A_{n}\right)\right)$

$=\left(a_{1}+\gamma_{2}\left(\mathrm{~A}_{1}\right)+\cdots+a_{n}+\gamma_{2}\left(A_{n}\right)\right) \in^{\mathrm{A}_{1}} / \gamma_{2}\left(\mathrm{~A}_{1}\right) \oplus \cdots \oplus^{\mathrm{A}_{n}} / \gamma_{2}\left(A_{n}\right)$.

Therefore by (2.2) and (2.3), we prove the equality (2.1).

Proposition 2.2. Let $\mathrm{A}_{1}, \ldots, A_{n}$ be a finite number of parafree Lie algebras and $R=\mathrm{A}_{1} * \cdots * A_{n}$ be the free product of algebras $\mathrm{A}_{1}, \ldots, A_{n}$. Then

$$
\mathrm{R} / \gamma_{2}(\mathrm{R})=\mathrm{A}_{1} / \gamma_{2}\left(\mathrm{~A}_{1}\right) \oplus \cdots \oplus^{\mathrm{A}_{n}} / \gamma_{2}\left(A_{n}\right)^{.}
$$

Proof. Let $u \in R$ and consider a homomorphism defined as

$$
\begin{gathered}
\sigma: R \rightarrow \mathrm{A}_{1} \oplus \cdots \oplus \mathrm{A}_{n} / \gamma_{2}\left(\mathrm{~A}_{1}\right) \oplus \cdots \oplus \gamma_{2}\left(\mathrm{~A}_{n}\right) \\
u+\gamma_{2}(\mathrm{R}) \rightarrow u+\gamma_{2}\left(\mathrm{~A}_{1}\right)+\cdots+\gamma_{2}\left(\mathrm{~A}_{n}\right) .
\end{gathered}
$$

By the definition of $\sigma$, it is clear that $\operatorname{Ker} \sigma=\gamma_{2}(\mathrm{R})$ and $\sigma$ is a surjective homomorphism. By isomorphism theorems we have

$$
\mathrm{R} / \gamma_{2}(\mathrm{R}) \cong \mathrm{A}_{1} \oplus \cdots \oplus \mathrm{A}_{n} / \gamma_{2}\left(\mathrm{~A}_{1}\right) \oplus \cdots \oplus \gamma_{2}\left(\mathrm{~A}_{n}\right)
$$

Therefore by the equality (2.1), we have

$$
\mathrm{R} / \gamma_{2}(\mathrm{R}) \cong \mathrm{A}_{1} / \gamma_{2}\left(\mathrm{~A}_{1}\right) \oplus \cdots \oplus^{\mathrm{A}_{n} / \gamma_{2}\left(A_{n}\right)}
$$

Lemma 2.1. Let $R=\mathrm{A}_{1} * \cdots * A_{n}$ be a parafree Lie algebra. Then the free factors $\mathrm{A}_{1}, \cdots, A_{n}$ are parafree.

Proof. We want to prove that for $i=1, \ldots, n$ the algebras $A_{i}$ are residually nilpotent and each one has the same lower central sequence as a free Lie algebra. Since $\mathrm{R}$ is parafree then it is residually nilpotent. Therefore for $i=1, \ldots, n$, algebras $A_{i}$ are residually nilpotent [Velioğlu, 2013].

Now by the Proposition 2.2, we have 


\section{International Advanced Research Journal in Science, Engineering and Technology}

Vol. 7, Issue 2, February 2020

$$
\mathrm{R} / \gamma_{2}(\mathrm{R}) \cong \mathrm{A}_{1} / \gamma_{2}\left(\mathrm{~A}_{1}\right) \oplus \ldots \oplus^{\mathrm{A}} \mathrm{A}_{\gamma_{2}}\left(A_{n}\right)
$$

For $i=1, \ldots, n$, let sets $X_{i}$ be generating sets of Lie algebras $\mathrm{A}_{i}$ such that $X_{i}$ are linearly independent modulo $\gamma_{2}\left(\mathrm{~A}_{i}\right)$. Therefore by Proposition 2.2, the set $X_{1} \cup \cdots \cup X_{n}$ is linearly independent modulo $\gamma_{2}(\mathrm{R})$ and it freely generates parafree Lie algebra $\mathrm{R}$ modulo $\gamma_{2}(\mathrm{R})$. Also we know that for $i=1, \ldots, n$ the sets $X_{i}$ freely generate Lie algebras $\mathrm{A}_{i}$ modulo $\gamma_{2}\left(A_{i}\right)$.

On the other hand quotient algebra $\mathrm{R} / \gamma_{\mathrm{n}}(\mathrm{R})$ is a free nilpotent Lie algebra that freely generated by the set $X_{1} \cup \cdots \cup$ $X_{n}$. Hence by Bahturin (1978), Theorem 9 , for $i=1, \ldots, n$, the sets $X_{i}$ generate a free nilpotent Lie algebra modulo $\gamma_{\mathrm{n}}\left(\mathrm{A}_{i}\right)$. Therefore each Lie algebra $A_{i}$ has the same lower central sequence as a free Lie algebra. Finally we have that the free factors $A_{i}$ are parafree.

\section{REFERENCES}

[1]. G. Baumslag, 'Groups with the same lower central sequence as a relatively free group I. The groups', Trans. Amer. Math. Soc., 129, 308-321, 1967.

[2]. G. Baumslag, 'Groups with the same lower central sequence as a relatively free group. II Properties', Trans. Amer. Math. Soc., 142, 507-538, 1969.

[3]. H. Baur, 'Parafreie Lie algebren und Homologie', Diss. Eth Nr., 6126, 1978.

[4]. K. Erdmann, MJ Wildon, "Introduction to Lie algebras", Springer, 2006.

[5]. N. Ekici, Z.Velioğlu, 'Unions of Parafree Lie Algebras", Hindawi Publishing Corporation Algebra, Vol. 2014, ID: 385397,1-5, 2014

[6]. N. Ekici, Z. Velioğlu, 'Direct Limit of Parafree Lie Algebras', Journal of Lie Theory, 25(2), 477-484, 2015.

[7]. Y. Bahturin, "Identical Relations in Lie Algebras', VNU Science Press, Utrecht, 1987.

[8]. Z. Velioğlu, 'Direct Sum and Free Factors of Parafree Lie Algebras, International Journal Pure \& Applied Mathematics',83(4),601-606, 2013.

[9]. Z. Velioğlu, 'Subalgebras and Quotient Algebras of Parafree Lie Algebras', International Journal Pure and Applied Mathematics, 83(3),507514, 2013.

[10]. Z.Velioğlu, N.Ekici, '’Paraserbest Lie Cebirlerinin Metabelyen Çarpımı, 2-Simetrik Kelimeler ve Verbal Alt Cebir', Erzincan Üniversitesi Fen Bilimleri Dergisi, 12(2),771-777, 2019.

[11]. Z.Velioğlu, 'Parafree Metabelian Lie Algebras which are Determined by Parafree Lie Algebras', Communications Faculty Sciences University of Ankara Series, A1:Mathematics and Statistics, 68(1),883-888, 2019. 